\title{
The Timing of erf-Mediated Recombination in Replication, Lysogenization, and the Formation of Recombinant Progeny by Salmonella Phage P22
}

\author{
STEVEN WEAVER ${ }^{1}$ AND MYRON LEVINE ${ }^{2}$
}

\author{
Department of Human Genetics, The University of Michigan, Ann Arbor, Michigan 48109
}

Accepted August 12, 1976

\begin{abstract}
Following infection, the development of phage P22 by either the lytic or the lysogenic pathways requires recombination, mediated either by the phage erf system or by the bacterial rec system [Botstein, D., and Matz, M. J. (1970) J. Mol. Biol. 54, 417-440]. We have investigated the timing of the essential recombinational processes with temperature-shift experiments using a temperature-sensitive erf mutant. In $\mathrm{rec}^{-}$cells, erf function appears to be required early in the infection to complete some essential step, the timing of which is the same in both the lytic and lysogenic circumstances. Once the step has taken place, subsequent development can occur without further erf function. However, the bulk of recombinant progeny arising in lytic crosses in rec- cells result from nonessential erf action late in the infection, after the time of the required early step.
\end{abstract}

\section{INTRODUCTION}

Phage P22 DNA is derived from a concatemeric precursor (Botstein, 1968; Botstein and Levine, 1968). The mature DNA molecules are linear, circularly permuted, and possess terminally redundant ends (Rhoades et al., 1968; Tye et al., 1974). Lytic replication by this phage must provide the means by which a concatemeric form can be constituted starting from a single terminally redundant parental DNA molecule. Two models have been proposed for achieving this. A long multimer could be assembled by a series of recombination events between homologous ends of shorter molecules (Streisinger et al., 1967). Alternatively, recombination between the redundant ends of a single DNA molecule would generate a circular form which could give rise to a concatemer by replication in a rolling circle mode (Gilbert and Dressler, 1968). Lysogenization requires that the phage chromosome be circularized prior to its integration as prophage

' Present address: Department of Bacteriology and Immunology, The University of North Carolina, Chapel Hill, North Carolina 27514.

${ }^{2}$ Author to whom reprint requests should be addressed.
(Campbell, 1962). At the crux of these hypotheses are recombination events, either inter- or intramolecular, involving the terminal redundancies.

These considerations suggest an essential role for recombination in both lytic and lysogenic pathways of P22 development. This idea was reinforced by the isolation of phage mutants that can neither replicate normally in nor lysogenize recombination-deficient hosts on infection (Botstein and Matz, 1970; Yamagami and Yamamoto, 1970). These mutants can, however, lysogenize and replicate in $\mathrm{rec}^{+}$ cells, although not so well as wild-type P22. Mutants possessing this phenotype are called erf mutants, since they are apparently defective for some essential recombination function. The failure to grow lytically is not absolute; some $10 \%$ of $\mathrm{rec}^{-}$ cells infected with erf- phage yield a small, variable number of progeny. This fact makes a measurement of recombination frequencies possible. Botstein and Matz (1970) found that in $\mathrm{rec}^{-}$hosts, removal of erf function results in a 5- to 20fold reduction in recombination frequencies, as well as a 100 -fold reduction in overall phage production. 
While erf mutants are by themselves unable to lysogenize $\mathrm{rec}^{-}$hosts, stable rec(erf $\left.{ }^{-} c 2-t s\right)$ lysogens can be constructured by complementation. On thermal induction of these lysogens, phage production ensues normally. The defect in growth seen on infection is not manifested on induction (Botstein and Matz, 1970). Since the prophage probably is excised from the host chromosome as a circle (Campbell, 1962), and since the generalized recombination functions erf and rec seem to be dispensable on induction, Botstein and Matz (1970) argued that the defect in both growth and lysogenization following infection reflects the failure of the linear parental DNA to be circularized via recombination at the terminal redundancies.

As discussed above, a substantial proportion of genetic recombination in P22 infections of $\mathrm{rec}^{-}$cells can be attributed to erf function. Yet circularization itself, being an intramolecular event, cannot be directly responsible for recombination between genetic markers. On this basis, we surmised that the generalized recombinational activity of the erf product might be distinguishable from its essential role in the lytic and lysogenic pathways of phage development. The availability of a temperature-sensitive erf mutant enabled us to test and extend these ideas. Using temperature-shift experiments, we investigated the timing of erf function with respect to lytic growth, lysogenization, and recombination.

\section{MATERIALS AND METHODS}

Bacteria and phage. Table 1 lists the bacterial strains used in this work; all are derivatives of Salmonella typhimurium LT2. Wild-type phage P22 and the two clear mutants, $c 1-7$ and $c 2-5$, have been described (Levine, 1957; Levine and Curtiss, 1961). Botstein and Matz (1970) isolated and mapped the erf 1 and erf 4 mutations. The ts 65 mutant was obtained from N. Yamamoto. The ts6.1 mutation was isolated in this laboratory and first mapped by Gough and Levine (1968). Levine and Schott (1971) described the 18-ts 18.1 mutation. Two amber mutations serve in this study as genetic markers: 18-am $\mathrm{H} 100$ (Bode et al., 1973) and 16-amN121 (Bot-
TABLE 1

Bacterial Strains

\begin{tabular}{|c|c|c|c|c|}
\hline $\begin{array}{l}\text { Designa- } \\
\text { tion }\end{array}$ & $\begin{array}{c}\text { Relevant gen- } \\
\text { otype }\end{array}$ & $\begin{array}{l}\text { Syn- } \\
\text { onym }\end{array}$ & \multicolumn{2}{|c|}{ Source or reference } \\
\hline 18 & Wild type & & Levine (1957) & \\
\hline 325 & $s u_{a m 19}^{+}$ & DB74 & $\begin{array}{c}\text { Botstein and } \\
(1970)\end{array}$ & Matz \\
\hline 330 & $\operatorname{rec} \mathrm{A}^{-}$ & DB47 & $\begin{array}{c}\text { Botstein and } \\
(1970)\end{array}$ & Matz \\
\hline 334 & $s u_{a m 19}^{+} r e c A^{-}$ & DB77 & $\begin{array}{r}\text { Botstein and } \\
(1970)\end{array}$ & Matz \\
\hline
\end{tabular}

stein et al., 1973). Phage-carrying combinations of these mutations were constructed by standard recombinational techniques.

Media. LB broth (Levine, 1957) was used for all liquid cultures. Dilutions of phage were made in buffered saline (Botstein, 1968). Tryptone agar plates, used for routine plating of phage and bacteria, are described by Bronson and Levine (1971). Green indicator agar (Bresch, 1953; Levine and Curtiss, 1961) and soft agar for overlays (Levine, 1957) have been described.

Preparation and assay of phage stocks. An overnight culture of an appropriate host was diluted 10-fold in LB broth and inoculated with phage from a single fresh plaque. After incubation for $3 \mathrm{hr}$ at $37^{\circ}$ or for 5 to $6 \mathrm{hr}$ at $25^{\circ}$, the cultures were treated with chloroform, and surviving cells and debris were removed by lowspeed centrifugation. Phage were assayed by the agar overlay method. Stocks prepared in this way usually exceed $10^{11} \mathrm{PFU} /$ ml. Titers are stable for several years without further purification or concentration.

Standard conditions of infection. Logphase cells at a concentration of $10^{8} / \mathrm{ml}$ were used in all cases. For infections carried out at $37^{\circ}$ or higher, phage were allowed to adsorb for $5 \mathrm{~min}$. Unadsorbed phage were then inactivated by exposure to anti-P22 serum (final $K=2$ per minute) for an additional $5 \mathrm{~min}$, followed by dilution of at least 1:1000 into serum-free medium. For some purposes it was necessary to initiate the infection at $25^{\circ}$. In these cases, the adsorption period and serum treatment were each extended to $10 \mathrm{~min}$.

Streak tests for immunity. Colonies to be tested were streaked with sterile tooth- 
picks across a line of $c 2-5$ phage (concentration, $10^{10} / \mathrm{ml}$ ) on indicator agar. Any darkening or interruption of the bacterial streak is indicative of sensitivity to superinfection. Only completely light, uniform streaks were scored as stable lysogens.

Treatment of lysates with base-plate protein. Lysates of P22 grown at $37^{\circ}$ or above occasionally contain phage that are unable to adsorb because of a deficiency of base-plate protein. These particles can be reconstituted to active phage in vitro by incubation in the presence of purified baseplates, or "tails" (Israel, 1967). To eliminate this potential source of variation, all high-temperature lysates were incubated with excess base-plate protein, which was purified as described by Hoffman and Levine (1975) before being assayed.

\section{RESULTS}

\section{Mapping and Complementation of erf Mutants}

The $t s 65$ and $t s 6.1$ mutations confer the same plating phenotype as the erf mutations described by Botstein and Matz (1970). At the nonpermissive temperature they fail to plate on $\mathrm{rec}^{-}$lawns and make small plaques with reduced efficiency on $\mathrm{rec}^{+}$indicators. To establish that these two mutations are in fact erf alleles, complementation between these mutants and known erf mutants under nonpermissive conditions was tested in liquid culture, as described in the legend to Table 2. Pairwise co-infection of $\mathrm{rec}^{-}$cells with $t \mathrm{s65}$, ts6.1, or erf 2 phage at high temperature results in a burst size no higher than that obtained in the case of single infections (Table 2, lines 11-13 and 8-10). However, both $t s 65$ and $t s 6.1$ are able to complement phage carrying a mutation in another early gene, 18-ts 18.1, with good efficiency (lines 5-6 and 1-3). We conclude that $t s 65$ and $t s 6.1$ are mutations in the erf gene.

The erf-ts6.1 mutation maps just to the left of the unconditional mutations erf 4 and erf 1 (Botstein and Matz, 1970). Three factor crosses were done to establish the position of the erf-ts 65 site relative to the other erf $^{-}$loci, using the clear mutations c1-7 and c2-5 as unselected outside markers (Table 3). In the first cross, erf 4 c1
TABLE 2

Complementation Between erf MUtants ${ }^{a}$

\begin{tabular}{rlr}
\hline Infection & \multicolumn{1}{c}{ Phage } & Burst size \\
\hline \multicolumn{3}{c}{ Experiment I } \\
1 & $t s 65$ & 1.5 \\
2 & $t s 6.1$ & 2.5 \\
3 & $t s 18.1$ & 0.01 \\
4 & $t s 65 \times t s 6.1$ & 1.7 \\
5 & $t s 65 \times t s 18.1$ & 85 \\
6 & $t s 6.1 \times t s 18.1$ & 115 \\
7 & $c 2-5$ & 400 \\
& & \\
& Experiment II & \\
8 & $t s 65$ & 1.9 \\
9 & $e r f 2$ & 3.6 \\
10 & $t s 6.1$ & 3.6 \\
11 & $t s 65 \times e r f 2$ & 2.5 \\
12 & $t s 65 \times t s 6.1$ & 2.6 \\
13 & $e r f 2 \times t s 6.1$ & 3.5 \\
14 & $c 2-5$ & 250 \\
\hline
\end{tabular}

${ }^{a}$ The rec $\mathrm{A}^{-}$strain 330 was infected at a total multiplicity of 10 and incubated for $100 \mathrm{~min}$ at $41^{\circ}$. The cultures were then shaken with chloroform, and the lysates were treated with base-plate protein and assayed. All phage in this experiment carried the c2-5 mutation. The burst sizes, expressed as progeny per infected cell, have been corrected for unabsorbed phage.

$\times$ erf-ts65, most of the rf $^{+}$recombinants carry the $c^{+}$morphology marker, indicating that erf-ts65 maps to the left of erf4. Similarly, the results of the second cross, erf 1 c $1 \times$ erf-ts 65 , place the erf-ts 65 locus to the left of the erf 1 mutation. In the third cross, erf-ts $65 c 2 \times$ erf-ts6.1, most of the recombinants carry the morphology determinant of the erf-ts6.1 parent, demonstrating that the erf-ts65 locus lies to the right of erf-ts6.1. These data suffice to place the erf-ts65 site with respect to the erf mutations mapped by Botstein and Matz (1970). The overall order is

$$
\text { erf-ts6.1 - erf-ts65 - erf } 4 \text { - erf } 1-c .
$$

\section{Replicational Requirement for erf}

By manipulating the incubation temperature of a rec- culture infected with erf-ts phage, one can restrict the activity of the erf gene product to given time intervals. From the consequences of such manipulations, inferences may be drawn about the timing of erf function vis à vis replication, 
lysogenization, and genetic recombination.

The timing of the replicational requirement for erf was addressed by a cold-pulse experiment. A culture of rec $\mathrm{A}^{-}$cells (strain 330) was infected with erf-ts65 c2-5 phage at $39^{\circ}$, the nonpermissive temperature. At 1-min intervals, small aliquots of the culture were chilled for $2 \mathrm{~min}$ at $25^{\circ}$, the permissive temperature, and then returned to $39^{\circ}$ until 120 min after infection. The relationship between burst size and time of low-temperature incubation, or "cold-pulse," is shown in Fig. 1.

Two-minute cold pulses begun as late as 5 min after infection result in a burst size of only 2 to 3 phage per cell, no higher than the burst of 2.5 obtained with a completely nonpermissive control infection. However, low-temperature exposure just 1 or $2 \mathrm{~min}$ later (from 6 to 8 or from 7 to 9 min after infection) allows a substantial increase in phage yield, to about 15 phage per cell. Cold pulses administered from the 8th minute on are less permissive, allowing a burst of only 6 to 8 phage per cell. Although below the optimal level of 15 effected by the 7- to 9-min pulse, these yields are nonetheless significantly above those seen under nonpermissive conditions or with very early pulses.

These data indicate a replicational requirement for erf function soon after infection, roughly between the 6 th and 9 th minute at $39^{\circ}$. The burst size that results when an erf-ts infection is cold-pulsed for $2 \mathrm{~min}$ during this period, 15 phage per cell, is well below the burst size of 105 observed for the permissive control infection. This difference is probably due in part to some asynchrony among the infected cells, together with the shortness of the cold pulse. Two minutes may be too brief a period to allow for completion of the essential step in more than a minority of infected cells. This contention is supported by our finding that a 3-min cold pulse administered from the 6 th to the 9 th min after infection yields a burst of some 40 per cell (data not shown). Unfortunately, the resolution of an optimal time for erf function is reduced with 3-min cold pulses.

\section{Timing of the Replicational and Recombi- national Requirements for erf Activity}

The results of the foregoing experiment suggest that the product of the erf gene functions early in infection to permit repli-

TABLE 3

MAPPING THE erf-ts65 MUtation ${ }^{a}$

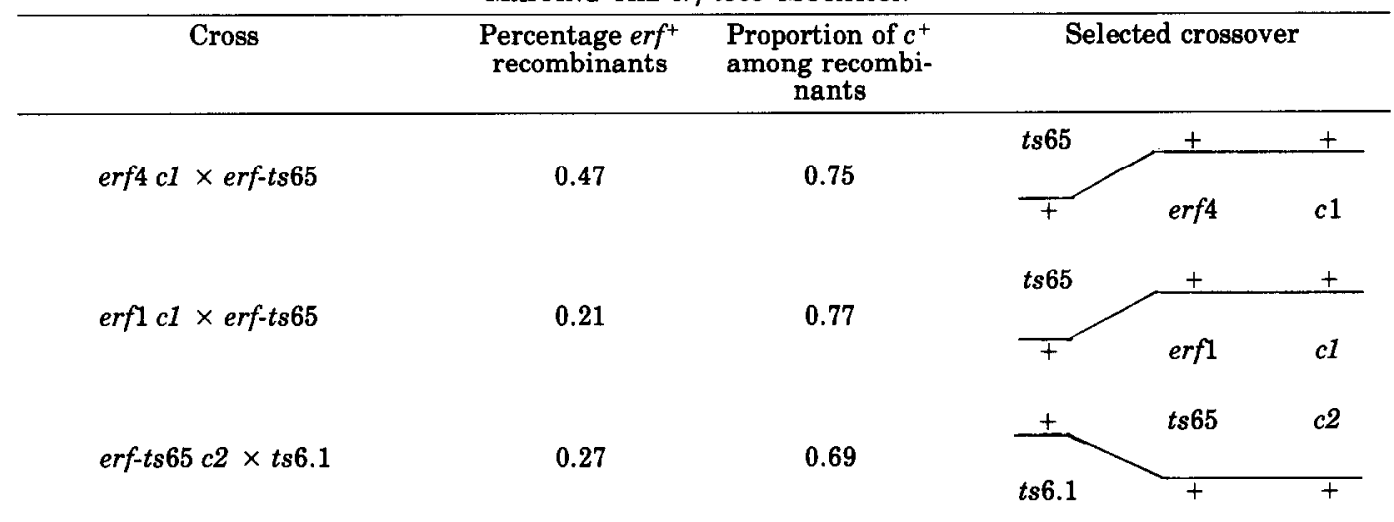

Overall gene order: ts6.1 erf-ts 65 (erf 4 erf1) $c$

\footnotetext{
a Infections were carried out in strain 18 at $25^{\circ}$ as described in Materials and Methods. The multiplicity of infection was 10 for each parent. Total phage progeny was assayed at $25^{\circ}$ using strain 18 as indicator; erf $f^{+}$ recombinants were scored at $37^{\circ}$ on strain 330 lawns. The plaque morphology types were scored in both cases. The proportion of $\mathrm{c}^{+}$among the $\mathrm{erf}^{+}$recombinants, listed above, is corrected for the proportion among the total progeny of the cross. The map order given for the erf 4 and erf1 loci was established by Botstein and Matz (1970).
} 


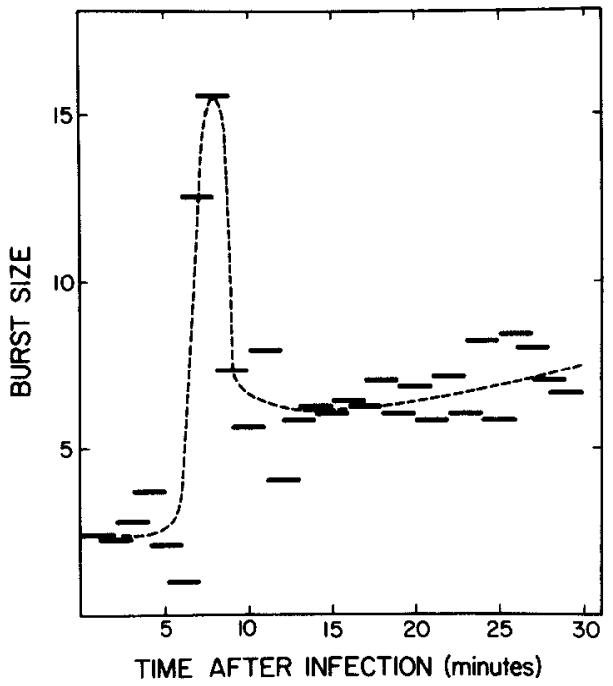

FIG. 1. Cold-pulse experiment. Strain 330 ( $\left.r e c \mathrm{~A}^{-}\right)$cells were infected at $39^{\circ}$ with erf-ts65 c2-5 phage at a multiplicity of 10 . Portions of the culture were chilled briefly at intervals in the manner described in the text. The conditions of infection are as given in Materials and Methods, except that the serum treatment was administered $30 \mathrm{~min}$ after infection. After $120 \mathrm{~min}$ of incubation, the cultures were treated with chloroform and base-plate protein and assayed on strain $18\left(\mathrm{rec}^{+}\right)$at $25^{\circ}$. The data are expressed as phage per infected cell. The horizontal bars indicate the timing of each cold pulse. A control infection carried out entirely at $25^{\circ}$ yielded 105 phage per cell; completely nonpermissive conditions $\left(39^{\circ}\right)$ led to a burst size of 2.5 .

cation to proceed, but give no information about the generalized recombinational processes that are also associated with erf activity. We pursued this matter with an experiment to measure the effect on recombination of limiting the function of erf to the early stages of infection, long enough to permit some replication, and then blocking its action for the balance of the latent period. The experimental design, then, was to initiate an erf-ts infection at $25^{\circ}$, to transfer aliquots of the culture to the nonpermissive temperature at various times, and to determine the consequences of temperature shift on the frequency of recombination, as well as on the overall yield of phage.

A cross was initiated by mixedly infecting strain 334 cells $\left(r e c \mathrm{~A}^{-} s u^{+}\right)$at $25^{\circ}$ with erf-ts65 c2-5 phage marked with different amber mutations. Half of the parental phage carried the gene 16 mutation $a m \mathrm{~N} 121$, and half carried the gene 18 marker $a m \mathrm{H} 100$. The total multiplicity was 20 . Immediately after the addition of phage, and every few minutes thereafter, a portion of the culture was shifted up to $41^{\circ}$. All samples were incubated for 240 min (the latent period is about $200 \mathrm{~min}$ at $25^{\circ}$ under these conditions), treated with chloroform and base-plate protein, and assayed for total progeny on strain 325 and for $\mathrm{am}^{+}$recombinants on strain 18 . Since the cross was carried out in an amber suppressing host, the amber mutations serve only as genetic markers; the frequency of recombination between them is determined from the proportion of $\mathrm{am}^{+}$progeny among the phage yield. To control for nonspecific effects of temperature shift, either on replication or recombination, a second cross was performed. In this case, the parental phage carried the same amber markers but were $\mathrm{erf}^{+}$. The data obtained from the two crosses, carried out in parallel, are presented in Fig. 2.

The effects of temperature shift on overall phage replication will be considered first. Shifting up an erf-ts $c 2$ infection at any time before about the 17 th min leads to an essentially nonpermissive situation (Fig. 2A). The low burst size observed, about one progeny phage per cell, is characteristic of erf $^{-}$infections of $\mathrm{rec}^{-}$cells (Botstein and Matz, 1970, and Table 2). However, if the infected culture is allowed to remain at low temperature for the first $30 \mathrm{~min}$ after infection, the resulting yield of phage is much higher, some 25 phage per cell. Since this effect of temperature is not observed in the erf $f^{+}$situation (Fig. $2 \mathrm{~B}$ ), it can be concluded that replication in $\mathrm{rec}^{-}$cells will be defective unless the product of the erf gene carries out some essential activity between roughly the 15 th and 30 th min after infection at $25^{\circ}$.

In this experiment, completion of the essential step allowed a burst of 25 progeny per cell; in similar experiments a yield of as many as 50 phage per cell has been observed with cultures shifted to high temperature at $30 \mathrm{~min}$ (data not shown). These levels are still somewhat below the yield seen with the low-temperature con- 


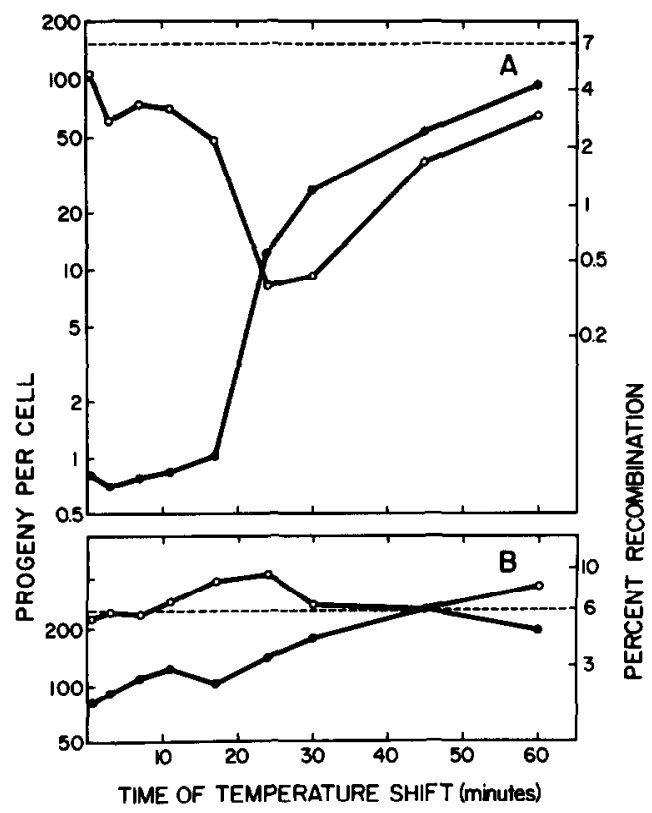

Fig. 2. Effect of temperature shift on recombination and replication. Strain $334\left(\mathrm{recA} \mathrm{A}^{-} \mathrm{su}^{+}\right)$cells infected with a mixture of $16-a m N 121$ and $18-a m$ H100 phage were shifted from 25 to $41^{\circ}$ at various times after infection, as described in the text. Panel A shows the results of the cross involving erf-ts 65 c2-5 parents; in Panel B the results of the $e r f^{+} c 2-5$ cross are shown. The curves describe the effects of temperature shift on total phage yield (- - - ) and recombination frequency (-O-). The ordinates are plotted on a logarithmic scale. The dashed horizontal lines indicate the yields and recombination frequencies observed for the two crosses when done under totally permissive conditions, i.e., at low temperatures.

trol infections, indicating that overall replication is somewhat temperature sensitive even late in infection, after the time of the essential erf activity. High-temperature exposure late in infection (after 30 min) consistently shows a slight inhibitory effect on phage production. This inhibition is somewhat more pronounced in the erf than in the erf $f^{+}$case.

The frequency of genetically recombinant phage is influenced by temperature shift in a more complex manner than is overall replication. When an erf-ts $c 2$ infected culture is shifted up at or before 17 min, a recombination frequency about half that of the permissive control infection is observed (Fig. 2A). However, if the culture is kept at $25^{\circ}$ for an additional $10 \mathrm{~min}$, the recombination frequency is lowered by a factor of 10 , to a level 20 -fold lower than the permissive control. After the 30th min, holding the culture at the permissive temperature for progressively longer periods brings about a steady increase in recombination. These two effects - the drop between the 15th and 25th min, and the rise after the 30th min-are not seen in the erf $^{+}$cross of Fig. 2B. This suggests that erf can act in ways that have opposite effects on the recombination frequency. If erf is only permitted to act early, up to the 25th min, the recombination frequency will be lower than the frequency that results when erf is not permitted to act at all. This is in contrast to the consequences of erf function after the 30th min. Late in infection, erf behaves as a generalized recombination function; the longer it is permitted to act, the higher is the final proportion of recombinant progeny. In this experiment, a recombination frequency 20 -fold lower than that of the permissive control resulted when the culture was shifted up at $30 \mathrm{~min}$. It follows that the majority of recombinants appear as a consequence of erf action late in infection, whereas erf exerts its greatest effect on overall replication earlier, during the 20 - to 30 -min interval.

Similar crosses have been performed with a number of marker pairs, with substantially the same results. The nonpermissive condition, which results from shifting up before the 15 th min, leads to a burst of 1 to 2 progeny per cell, and a recombination frequency two- to fivefold lower than the permissive control. Between 15 and 30 min after infection, the activity of erf makes the production of 25 to 50 phage per cell possible. However, the frequency of recombinants among these progeny is 5 to 10 times lower than the frequency among the few progeny that are produced under the nonpermissive condition. A 20 - to 40 -fold increase in the proportion of recombinant progeny results from erf action after the 30th min. During this period, erf function has at most a small effect on overall replication.

\section{Timing of erf Function in Lysogenization}

As mentioned in the Introduction, Botstein and Matz (1970) have suggested that 
the erf defect in both replication and lysogenization is the same: an inability to circularize the parental chromosome. If this is the case, one might expect that the timing of the requirement for $\operatorname{erf}$ would be the same in the lytic and lysogenic pathways of development. We tested this notion in the obvious way, by studying the effects of temperature shift on lysogenization of $\mathrm{rec}^{-}$ cells by erf-ts $65 c^{\prime}$ phage.

Strain 330 cultures were infected at low temperature with either erf-ts65 or wildtype phage. After $10 \mathrm{~min}$, the infected cells were diluted into medium containing antiphage serum. To avoid complications due to secondary infections, the surviving cells were kept in serum-containing medium from this point on. At 5-min intervals, portions of the two cultures were transferred to $39^{\circ}$. The process of integration by wild-type P22 is inherently temperature sensitive and takes place late in the infection, after cell division has resumed (Smith and Levine, 1967). So, to encourage integration, all subcultures were shifted back to $25^{\circ}$ for $2 \mathrm{hr}$ starting at $60 \mathrm{~min}$. Then, at $180 \mathrm{~min}$, the survivors were moved back to $37^{\circ}$ (to encourage growth) and allowed to divide for an additional $5 \mathrm{hr}$ (about 10 generations) before being spread on plates in the presence of additional anti-phage serum. The colonies which developed were tested for immunity as described in Materials and Methods.

The frequencies of lysogenic survivors observed are plotted in Fig. 3. Clearly, lysogenization by erf-ts phage is strongly inhibited by high temperature early in infection. Virtually no lysogens were found in cultures shifted up at $20 \mathrm{~min}$ or before. Although the effect is less dramatic, lysogenization by wild-type phage was also reduced substantially by shifting to high temperature. To distinguish the general inhibition of lysogenization by high temperature from the effects attributable to the erf defect, the data obtained for the erf$t s$-infected cells were normalized to the results of the wild-type infection. For each time of shift, the immune fraction exhibited by survivors of the erf-ts infection was divided by the proportion of immune survivors of the wild-type infection. These ratios are plotted in Fig. 4. The timing of

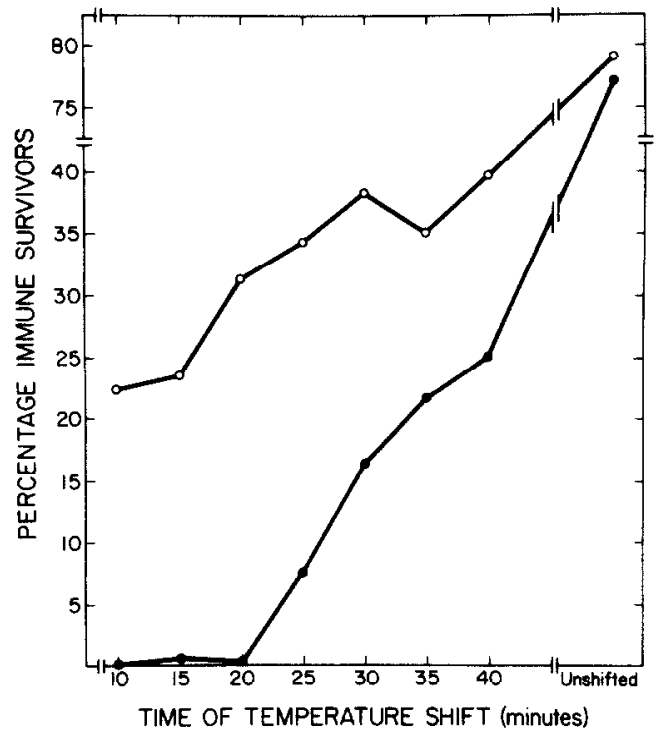

FIG. 3. Effect of temperature shift on lysogenization by erf-ts and erf ${ }^{+}$phage. Strain 330 (recA $\mathrm{A}^{-}$) cultures were infected a multiplicity of 20 . The incubation temperature was manipulated as described in the text. This figure shows the influence of temperature shift on the frequency of lysogens among the survivors of the erf-ts65 $c^{+}\left(\longrightarrow-{ }_{-}\right)$and erf $^{+} c^{+}$ $(-\mathrm{O}-$ ) infections.

erf function in the course of lysogenization is reminiscent of its effects in lytic development. If erf activity is blocked before the 20 th min, virtually none of the surviving cells will be lysogenic. After minute 20, erf makes possible the establishment of lysogeny in an increasing proportion of cells. If erf function is allowed to continue as long as the 40 th $\mathrm{min}$, lysogenization will be two-thirds as efficient as in the wild-type situation. Under completely permissive conditions, erf-ts mutants lysogenize exactly as well as wild-type phage.

\section{DISCUSSION}

In both the lytic and lysogenic development of phage P22, there is an obligatory involvement of a recombination pathway. The requirement can be fulfilled either by the action of the bacterial rec system or by the phage's own recombinational machinery, the erf system (Botstein and Matz, 1970; Yamagami and Yamamoto, 1970). The experiments that we have described reveal the timing of the essential action of the erf system on infection of $\mathrm{rec}^{-}$cells, as well as the timing of its involvement in the 


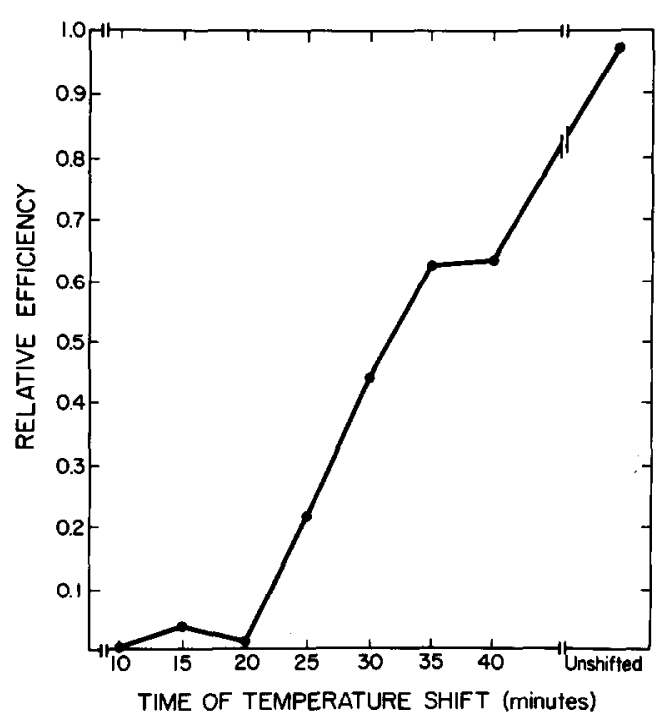

FIG. 4. Timing of erf function in lysogenization. The data of Fig. 3 are expressed in a different way. For each time of shift, the efficiency of lysogenization by the erf-ts infection is given as a fraction of the efficiency shown by the erf $f^{+}$infection.

formation of recombinant progeny phage.

In the case of lytic infections, our results indicate that the activity of the erf system is required early to carry out an essential step, possibly a recombinational modification of the structure of the parental phage DNA, which is prerequisite to subsequent replication and maturation. Under our conditions, the required recombinational events take place roughly between the 6 th and 9 th min after infection at $39^{\circ}$ or between the 20th and 30th min at $25^{\circ}$. Once accomplished, the essential recombination event seems to be sufficient for the production of 25 to 50 phage per cell, even if subsequent erf function is prevented. Yet, blocking erf function after a situation permissive for replication has been established greatly reduces the frequency of recombinant progeny, to a level at least 20 times lower than that seen under permissive conditions. This indicates that P22 can replicate in a way that results in few recombinant progeny and that the high levels of recombination characteristic of normal P22 lytic growth result from recombinational processes which occur late in the infection and which are not absolutely required for replication and matura- tion. This is consistent with the idea that recombination in P22 development is required early for the circularization of the linear parental DNA (Botstein and Matz, 1970), since such an event cannot directly recombine genetic markers. The possibility that erf action late in infection is responsible for increasing the yield of progeny is not excluded by our results.

Even under nonpermissive conditions, a small number of phage are still produced. Some recombinational system is obviously operating under the conditions of erf and rec inhibition, since two-point recombination frequencies are only two- to fivefold lower than in the control $\mathrm{erf}^{+} \mathrm{rec}^{-}$condition. This slight inhibition, in the face of a defect severe enough to limit replication to an average of one to two phage per cell, suggests that the low-level growth could be dependent on the residual potential for recombination. Leakiness of either the rec or erf mutations might be responsible. Alternatively, a minor recombination pathway might be unmasked by the elimination of the major systems. Botstein and Matz (1970) showed by a single burst analysis that only about $10 \%$ of $\mathrm{rec}^{-}$cells in-

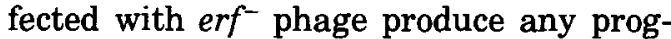
eny at all. The burst sizes are quite variable and average about 20 per productively infected cell. A trivial explanation for the limited replication that takes place despite the $\mathrm{erf}^{-}$and $\mathrm{rec}^{-}$constraints would be the mediation, by the residual recombination activity, of the essential event, presumably circularization, in a small fraction of the infected cells, allowing phage production then to take place. If so, the situation in these cells should be comparable to the situation achieved in a mass culture when recombination is blocked (by temperature shift) just after the time of the required erf step. One might then expect that similar levels of recombinant phage would be found among the progeny produced under these two conditions. This is not the case. In crosses such as that of Fig. 2A, the recombination frequencies seen under nonpermissive conditions, when the few progeny are produced by a minority of infected cells, are one-half to one-fifth the frequencies seen in the unshifted, permis- 
sive control cross. On the other hand, when erf is permitted to carry out its essential early function in all cells, but is then blocked for the remainder of the infection by a shift up at $30 \mathrm{~min}$, the frequency of recombination is reduced 20 - to 40 -fold relative to the control. This indicates that the trivial explanation proposed above is probably an oversimplification. Recombination may play a role in the limited replication that takes place under erf ${ }^{-}$ $\mathrm{rec}^{-}$conditions that is quite different from its essential role in normal replication.

DNA synthesis following infection by P22 c2 mutants occurs in two distinct stages (Smith and Levine, 1964). The first of these periods begins almost immediately after infection. At $37^{\circ}$, this early burst of synthesis peaks after about 5 to 6 min and then falls off rapidly. Some time later, at about the 16 th to 18 th min, late replication begins, continuing until lysis. In infections by $c 1$ mutants, the early period of synthesis occurs in the same way. However, the onset of late replication begins earlier, at about $10 \mathrm{~min}$ after infection. After infection by wild-type P22 under conditions favoring the lysogenic $r$ sponse, the early burst of synthesis takes place, but due to the establishment of repression, late replication is blocked. Botstein and Matz (1970) showed that the erf ${ }^{-}$ $\mathrm{rec}^{-}$recombination defect prevents late replication in lytic infections, but apparently has no effect on the amount or timing of the early peak of DNA synthesis. Taken together, these facts suggest that the essential erf step must take place before the 10th $\mathrm{min}$, the time at which late replication begins in $c 1$ infections. Our genetic results are consistent with this prediction. They show that the early step occurs between the 6th and 9th min after infection at $39^{\circ}$, or between the 20th and 30 th min at $25^{\circ}$.

In summary, our results lend support to the contention of Botstein and Matz (1970) that the requirement for recombination in the life cycle of P22 reflects the need for linear phage DNA to be circularized prior to either replication or integration. We have shown that the required recombination events take place at the same time in both lytic and lysogenic infections. We have also demonstrated that blocking erf function after the occurrence of the essential recombinational step in lytic infections of $\mathrm{rec}^{-}$cells results in extensive replication without concomitant high. levels of genetic recombination. In other words, the role of erf in the formation of genetically recombinant progeny can be distinguished from its essential role in replication. Still, these results are not critical tests of the circularization hypothesis. Direct physical evidence that the appearance of circular P22 DNA (Rhoades and Thomas, 1968) after infection of $\mathrm{rec}^{-}$cells is dependent on erf function is presented in the accompanying paper (Weaver and Levine, 1977).

\section{ACKNOWLEDGMENTS}

We are grateful to Mr. Joseph Bellestri for skilled assistance, and to Drs. Don Clewell, David Friedman, and Ethel Jackson for discussions and for criticism of the manuscript. We thank Dr. N. Yamamoto for the gift of the erf-ts 65 mutant. This work was supported by Public Health Service Grant GM 15419 from the National Institute for General Medical Sciences. One of us (S. W.) was a National Institutes of Health predoctoral fellow supported by Training Grant No. GM 00071 from the U. S. Public Health Service.

\section{REFERENCES}

Bode, W., Dopatka, H. D., and Prell, H. H. (1973). Functional classification of P22 amber mutants. Mol. Gen. Genet. 127, 341-347.

Botstein, D. (1968). Synthesis and maturation of phage P22 DNA: I. Identification of intermediates. J. Mol. Biol. 34, 621-641.

Botstein, D., and Levine, M. (1968). Synthesis and maturation of phage P22 DNA: II. Properties of temperature-sensitive phage mutants defective in DNA metabolism. J. Mol. Biol. 34: 643-654.

Botstein, D., and MATz, M. J. (1970). A recombination function essential to the growth of bacteriophuge P22. J. Mol. Biol. 54: 417-440.

Botstein, D., Waddeli, C. H., and King, J. (1973). Mechanism of head assembly and DNA encapsulation in Salmonella phage P22. J. Mol. Biol. 80, $669-695$.

Bresch, C. (1953). Genetical studies on bacteriophage T1. Ann. Inst. Pasteur 84, 157-163.

Bronson, M. J., and Levine, M. (1971). Virulent mutants of bacteriophage P22. J. Virol. 7, 559568.

Campbell, A. (1962), Episomes. Advan. Genet. 11, 101-145.

Gilbert, W., and DRessler, D. (1968). DNA replica- 
tion: The rolling circle model. Cold Spring Harbor Symp. Quant. Biol. 33, 473-484.

Gough, M., and Levine, M. (1968). The circularity of the phage P22 linkage map. Genetics 58, 161169.

Hoffman, B., and Levine, M. (1975). A phage P22 virion protein which performs an essential early function: I. Analysis of $16^{-}$ts mutants. J. Virol. 16, 1536-1546.

ISRAEL, V. (1967). The production of inactive phage P22 particles following induction. Virolugy 33, 317-322.

Levine, M. (1957). Mutations in the temperate phage P22 and lysogeny in Salmonella. Virology 3, 22-41.

Levine, M., and Curtiss, R. (1961). Genetic fine structure of the $\mathrm{C}$ region and the linkage map of phage P22. Genetics 46, 1573-1580.

Levine, M., and Schotт, C. (1971). Mutations of phage P22 affecting phage DNA synthesis and lysogenization. J. Mol. Biol. 62, 53-64.

Rhoades, M., Machatrie, L. A., and Thomas, C. A., JR. (1968). The P22 bacteriophage DNA molecule: $\mathrm{I}$. The mature form. J. Mol. Biol. 37, 21-40.
Rhoades, M., and Thomas, C. A. (1968). The P22 bacteriophage DNA molecule: II: Circular intracellular forms. J. Mol. Biol. 37, 41-61.

Smith, H. O., and Levine, M. (1964). Two sequential repressions of DNA synthesis in the establishment of lysogeny by phage P22 and its mutants. Proc. Nat. Acad. Sci. USA 52, 356-363.

Smith, H. O., and Levine, M. (1967). A phage P22 gene controlling integration of prophage. Virology 31, 207-216.

Streisinger, G., Emrich, J., and Stahl, M. M. (1967). Chromosome structure in phage T4. III. Terminal redundancy and length determination. Proc. Nat. Acad. Sci. USA 57, 292-295.

Tye, B.-K., Huberman, J. A., and Botstein, D. (1974). Non-random circular permutation of phage P22 DNA. J. Mol. Biol. 85, 501-532.

Weaver, S., and Levine, M. (1977). Recombination circularization of Salmonella phage P22 DNA. Virology 76, 29-38.

YAMAGAMI, H., and Yamamoto, N. (1970). Contribution of the bacterial recombination function to replication of bacteriophage P22. J. Mol. Biol. 53, 281-285. 